\title{
Qizhi Decoction may Protect Diabetic Nephropathy Through Decrease Lipid and Inflammation
}

\author{
Cunyun Min*, Tingting Fu, Xuhui Huang, Yu Du, Changjun Wang \\ The Integrated Division of Chinese and Western Medicine, Guangdong Academy of Medical Sciences, Guangdong General Hospital, \\ Guangdong Academy of Geriatrics, Guangzhou, China
}

Email address:

mcy1288@163.com(Cunyun Min), mcy1288@outlook.com(Cunyun Min)

${ }^{*}$ Corresponding author

To cite this article:

Cunyun Min, Tingting Fu, Xuhui Huang, Yu Du, Changjun Wang. Qizhi Decoction may Protect Diabetic Nephropathy Through Decrease Lipid and Inflammation. International Journal of Chinese Medicine. Vol. 2, No. 2, 2018, pp. 6-13. doi: 10.11648/j.ijcm.20180202.11

Received: August 17, 2018; Accepted: September 12, 2018; Published: September 29, 2018

\begin{abstract}
Diabetic nephropathy (DN) affects approximately one-third of individuals with diabetes mellitus. Many patients develop kidney damage despite modern pharmacologic therapies available for DN treatment. Chinese medicine is a good choice for patients with DN. The aim of this study is to study the effect and safety of Qizhi decoction on patients with diabetic nephropathy. Approximately 255 men and women aged $\geq 18$ to $\leq 80$ years with DN were enrolled and randomized to two groups. The experimental intervention accepted Qizhi decoction in addition to their usual Novolin 50R penfill regimen. The control intervention accepted only Novolin 50R penfill. All participants receive their regimen for 12 weeks. Estimated glomerular filtration rate (eGFR), serum free fatty acid (FFA) level, the urine protein/creatinine ratio (U/C), plasma cystatin C (Cyst), Plasma C-reactive protein (CRP), hemoglobin A1c, serum uric acid, dyslipidemia were tested before entering the group. At the end of this study the index above and safety were measured again. Urine albumin levels, U/C, Cyst, CRP, FFA, TNF- $\alpha$ and MCP-1 levels decreased in both groups after 12 weeks of treatment. The eGFR, Cyst improved after12 weeks of treatment. Compared to the control, urine albumin levels, U/C, Cyst, CRP, FFA, TNF- $\alpha$ and MCP-1 levels decreased obviously in treatment group after 12 weeks of treatment. The kidney function, as measured by the eGFR, Cyst, significantly improved after 12 weeks of treatment. The results indicate that combined Chinese and western medicine should be the better choice of patients with DN. QZD may protect Diabetic nephropathy through decrease lipid and inflammation.
\end{abstract}

Keywords: Diabetic Nephropathy, Albumin, Lipid, Inflammation, Kidney Function, Chinese Herbs

\section{Introduction}

Diabetes represents one of the major health threats facing humans. The World Organization (WHO) has called diabetes the epidemic of the 21 st century. WHO estimated that more than 180 million people worldwide suffer from diabetes today and predicts that the number will double before 2030. Diabetic complications increase in severity and frequency as diabetes progresses. Almost $80 \%$ of patients with diabetes die from diabetic complications opposed to the poor control of plasma glucose levels. Diabetic nephropathy (DN) is one of the most relevant diabetic complications. Approximately $30-35 \%$ of patients with diabetes develop diabetic nephropathy despite modern pharmacologic therapies available for DN treatment. Even today there is no successful chemical therapy for diabetic nephropathy $[1,2]$.

It is the most frequent cause of end-stage renal disease (ESRD).

Hyperglycemia is critical in the genesis of diabetic complications. Poor glycemic control is an independent predictor of the development and progression of $\mathrm{DN}$, although the intimate mechanisms by which hyperglycemia leads to renal injury are not completely known [3]. Oxidative stress due to hyperglycemia is a major cause of diabetes complications. Hyperglycemia leads to an increase in oxidative stress by exacerbating glucose oxidation and mitochondrial generation of reactive oxygen species (ROS) which cause DNA damage and contributes to accelerated apoptosis. Hyperglycemia increases the expression of transforming growth factor-beta (TGF- $\beta$ ) in the glomeruli and of matrix proteins specifically stimulated by this cytokine. 
TGF- $\beta$ contributes to the cellular hypertrophy and enhanced collagen synthesis observed in persons with diabetic nephropathy [4]. Chronic hyperglycaemia results in both morphological and functional impairments of podocytes in the kidney. Investigation about the effects of high glucose $(\mathrm{HG})$ on the insulin signaling pathway suggests that $\mathrm{HG}$ compromises the insulin signaling pathway in the glomerulus, promoting a proapoptotic environment, with a possible critical step for this malfunction lying at the level of IRS-1 phosphorylation $[5,6]$.

In addition to high glucose, diabetes is associated with other metabolic derangements including protein, lipid and gaseous molecules such as, nitric oxide (NO) and hydrogen sulfide (H2S). Current evidence suggests that the pathogenesis of DN is multifactorial, and hyperglycemia mediates injury by several mechanisms such as fructokinase activation and ATP depletion, oxidative stress, production of inflammatory cytokines, activation of fibroblasts, and microaneurysm formation. Furthermore, imbalance of matrix metalloproteinases and their inhibitors leads to abnormal extracellular matrix metabolism, and disrupted gap junction proteins cause poor cell-cell communication [7].

Immunological and inflammatory mechanisms have been shown to have role in both the development and progression of diabetic nephropathy. Chronic inflammation plays an important role in the development of diabetes and its late complications. Macrophage accumulation is closely associated with chronic renal injury and progression. Macrophages are present in the glomeruli and interstitium of type 2 diabetic patients with DN and of controls. Although patients and controls had similar numbers of glomerular macrophages, glomerular anti-inflammatory CD163+ macrophages were associated with pathological lesions in DN. Macrophage depletion mediates renal tissue protection as proved by a reduction in albuminuria, histopathological changes, and kidney macrophage recruitment during diabetes [8]. Serum levels of IL-18 in nephropathic patient significantly rise and might be a predictor factor of progression of diabetic nephropathy. It is well recognized that proinflammatory NF-B is central in mediating signaling pathways that ultimately result in renal fibrosis and renal failure. TLR2 is likely to be the predominant long-term mediator of NF-B activation in transducing inflammation in diabetic nephropathy $[9,10]$.

The onset of diabetic nephropathy is characterised by a rise in albumin excretion rate (AER) and/or a transient rise in glomerular filtration rate (GFR). Moderately increased albuminuria is accepted as the first clinical sign of diabetic nephropathy. However, more and more morphometric studies and autopsy studies have demonstrated that by the time moderately increased albuminuria is evident, the kidneys in some diabetic patients have already undergone glomerular and tubulointerstitial damage, which indicates that it is not a sensitive marker for diabetic nephropathy [11, 12]. The estimated glomerular filtration rate (eGFR) in both groups of normal and microalbuminuric patients with type 2 diabetes decreased at follow-up compared to those at the baseline. The risk of annual eGFR decline rate $\geq 3 \mathrm{ml} / \mathrm{min} / 1.73 \mathrm{~m} 2$ increased as the baseline eGFR increased. Extra careful attention should be paid to patients with eGFR $\geq 120 \mathrm{ml} / \mathrm{min} / 1.73 \mathrm{~m} 2$ to detect cases with rapidly decreased GFR under the normal range. Multiple linear regression analysis using eGFR as the dependent variable demonstrated that uric acid (UA), FFA, triglyceride (TG), total cholesterol (TC), albuminuria, hypertension, smoking and duration of diabetes were all independent risk factors for decreased eGFR. A study suggests that the eGFRcre-cys equation may be more precise and sensitive for predicting the renal outcome in T2DN patients. Tracking renal decline using eGFRcre-cys may be used as a surrogate for determining the renal end point in a clinical setting. In established nephropathy, plasma cystatin $C$ based estimates of GFR are marginally superior to creatinine based estimates. The percentage change in cystatin $\mathrm{C}$, a recent new reliable marker for detecting subtle renal dysfunction, of $\geq 10 \%$ for $24 \mathrm{~h}$ after procedure is an independent predictor for developing DN $[13,14]$.

Current therapeutic approaches for diabetic nephropathy are focussing on blood pressure control with inhibitors of the renin-angiotensin-aldosterone system, on glycaemic and lipid control, and life-style changes. DN caused by hypertension and unmitigated inflammation in diabetics, renders the kidneys unable to perform normally, and leads to renal fibrosis and organ failure. The increasing global prevalence of DN has been directly attributed to rising incidences of Type II diabetes, and is now one of the largest non-communicable cause of death worldwide. Despite the high morbidity, successful new treatments for DN are lacking. Our research indicated that integreated Chinese medicine and western medicine is more effective than pure westen medicine [15]. The aim of this study is to eveluate the effect of integreated Chinese medicine and western medicine on patients with DN.

\section{Materials and Methods}

\subsection{Patients}

This is a prospective randomized clinical trial lasting 12 weeks. Patients were targeted for enrollment among stage III to sage IV diabetic nephropathy who regularly attended the Guangdong General Hospital for outpatient and inpatient departments treatment. The study enrolled 255 patients who meet the diagnostic criteria. They were numbered and grouped according to the staging criteria for DN. Then, the patients were assigned a zheng differentiation classification according to the traditional Chinese medicine (TCM) diagnostic criteria. All patients were randomly assigned to the treatment or control group using a random number table.

\subsection{Diagnostic Criteria}

Diagnostic criteria for DN (refer to ADA criteria [16])

Stage III: Urinary albumin excretion rate (UAER) falls within the range of 20 to $200 \mathrm{ug} / \mathrm{min}$ or 30 to $300 \mathrm{mg} / 24 \mathrm{~h}$, accompanied by slightly elevated blood pressure.

Stage IV: This stage is featured by a large amount of 
proteinuria, UAER $>200 \mathrm{ug} / \mathrm{min}$ or the urinary protein quantitative is persistently higher than $500 \mathrm{mg} / 24 \mathrm{~h}$, and it is nonselective proteinuria. Some may present elevated blood pressure and symptoms of nephrotic syndrome.

Traditional chinese medicine (TCM) standards (refer to criteria of "Guideline for TCM Diabetes Prevention and Treatment" of the China Association of Chinese Medicine [17].

The symptoms of Qi-yin asthenia include proteinuria, lassitude, shortness of breath, dizziness, dreaminess, frequent urination, palm and planter fever, palpitations, a thin and red (or pink) tongue, and a weak pulse. The signs of liver kidney yin insufficiency include proteinuria, vertigo, tinnitus, burning sensation of the five centers, soreness of the waist and knees, dry eyes, a red tongue, less moss, and a rapid pulse. The symptoms of Qi-blood asthenia include proteinuria, lassitude, shortness of breath, a pale or sallow complexion, dizziness, colorless lips and nails, palpitations, insomnia, soreness of the waist and knees, a pale tongue, and a weak pulse. The signs of spleen-kidney yang deficiency include proteinuria, mental fatigue, chills, swollen limbs (especially the lower limbs), a pale face, long or deficient urine, an increase in nocturia or diarrhea before dawn, a pale tongue with a fat body, and a slow and weak pulse.

\subsection{Inclusion Criteria}

Patients who met the Chinese and ADA diagnostic criteria were selected for the study.

\subsection{Exclusion Criteria}

Patients who do not meet the diagnostic criteria; those with kidney disease caused by other diseases; pregnant or lactating women; children; patients with other diseases or complications (e. g., congestive heart failure, elevated serum transaminase levels, primary hypertension); those with diabetic ketoacidosis or urinary tract infection within the previous month; those with other serious heart, brain, lung, liver and other primary organ diseases; those with malignant hypertension or myocardial infarction within the past 6 months; those with severe infections and other complications; those with a history of cerebrovascular accident; and those with a recent history of nephrotoxic drug use were excluded.

\subsection{Treatment}

Both groups completed 12-weeks of diet control and exercise therapy. The patients in the control group were routinely given gliquidone or Novolin 50R penfill to reduce blood glucose levels. Participants randomized to the treatment group would administer a daily dose of the Qizhi capsule. The medicine was decocted with water twice and taken twice after mixing.

Blood and urine specimens were collected before and after treatment. Participants were not allowed to take any other TCM, Chinese patent medicine to stimulate the liver or kidneys or to activate blood circulation, or other drugs that could interfere with the agents used in the study.

\subsection{Ethics Issue}

The study protocol was approved by the institutional review board at Guangdong General Hospital and conducted in accordance with the Declaration of Helsinki and its amendments. After a full explanation of the study, all patients gave written informed consent.

\subsection{Measurements}

\subsubsection{Laboratory Tests}

Before starting the study, all selected patients would undergo an initial screening assessment that include a medical history and physical examination. We would evaluate patients at the start of the study, then again after the 12-weeks of treatment.

Routine blood sampling to assess liver and kidney function as well as fasting plasma glucose (FPG), and 2-h postprandial glucose (2-hPG) levels. Duration of diabetes, age, systolic blood pressure, diastolic blood pressure, the presence of concomitant microvascular complications; and positive family history, elevated body mass index; smoking status; estimated glomerular filtration rate (eGFR); serum free fatty acid (FFA) level, creatinine, the urine protein/creatinine ratio (U/C), plasma cystatin C (Cyst), glutamic-pyruvic transaminase (ALT), glutamic-oxaloacetic transaminase (AST), hemoglobin A1c (HbA1c), albuminuria grade (ALBU), serum uric acid (SUA), $\beta$-2-microglobulin ( $\beta 2 \mathrm{MU})$, $\alpha$-1-microglobulin (a1MU), triglyceride (TG), low-density lipoprotein (LDL), total cholesterol (TC) and high-density lipoprotein cholesterol (HDL),. Plasma C-reactive protein (CRP) and so on were all evaluated.

\subsubsection{Measurement scale for TCM Symptoms}

The measurement scale for TCM symptoms recommended by the Guidelines for Clinical Research of Chinese Medicine (New Drug) [17] will be used for ease of assessment. Each of the primary symptoms necessary for the diagnosis of syndrome will be scored $0,2,4$ or 6 , while a secondary symptom is scored $0,1,2$ or 3 , and the scores then summed to yield a total score of both types of symptoms for a patient. It is stipulated that the total primary symptom score shall not exceed 18 , and the total score for secondary symptoms shall not exceed 33 for an individual patient.

\subsection{Efficacy Assessment Tools}

\subsubsection{For TCM Symptoms}

Following the Guidelines for Clinical Research of Chinese Medicine (New Drug) [17], the reduction in the total TCM symptom score of the patient will be calculated and used as an efficacy indicator (EI) for the evaluation of treatment efficacy. EIs will be calculated according to the following formula: EI = Total symptom score at baseline - Total symptom score post treatment / Total symptom score at baseline $\times 100 \%$. The degree of symptom improvement will be presented in four categories ranging from 'full recovery' (EI $\geq 90 \%$ ), 'good recovery' ( $90 \%>\mathrm{EI} \geq 70 \%)$, 'modest recovery' (70\% >EI $\geq 30 \%$ ) to 'no recovery' (EI $<30 \%$ ).

\subsubsection{For Laboratory Indicators of the Disease}

Full recovery: the quantitative 24-hour urinary protein, 
UAER and renal function return to normal levels. Good recovery: a decrease of $\geq 40 \%$ in UAER or the quantitative 24-hour urinary protein. Modest recovery: a decrease of $<40 \%$ in UAER or the quantitative 24-hour urinary protein. No recovery: unchanged or worsened laboratory test results.

\subsection{Reporting of Adverse Events}

Adverse events will be recorded in medical diagnostic terminology. Detailed symptoms, time of occurrence, duration, severity, possible causal relationships, actions taken, results and other relevant information will be reported. In the case of an adverse event, researchers must fill out a serious adverse event (SAE) form and notify both the institutional review board (IRB) and the regulatory authorities within 24 hours.

\subsection{Statistical Analysis}

Statistical analysis was conducted using Statistical Package for Social Sciences software version 19. 0. The data are presented as the mean $\pm \mathrm{S}$. E. Student's t-test was performed for comparisons between groups, and the $\chi^{2}$ test was used for numerical data. For all statistical analysis, $\mathrm{P}<0$. 05 was considered statistical significant.

\section{Results}

\subsection{Comparison of the Overall Efficacy of the Two Groups}

255 patients were enrolled in the study and of these 245 completed the study. The reason for premature withdrawal was lost-to-follow-up. The treatment group exhibited greater improvements in clinical symptoms and larger reductions in urinary protein levels $(\mathrm{P}<0.01)$. The combination therapy appeared to be more effective than Western medicine alone, as shown in Table 1.

Table 1. Comparison of the overall efficacy of the two treatments ( $[\%])$.

\begin{tabular}{|c|c|c|c|c|}
\hline & Clinical control & Markedly effective & Effective & Ineffective \\
\hline Treatment group & $40(29.4 \%)$ & $54(39.7 \%)$ & $27(19.85 \%)$ & $15(11 \%)$ 方 \\
\hline Control group & $26(23.85 \%)$ & $32(29.36 \%)$ & $21(19.27 \%)$ & $30(27.52 \%)$ \\
\hline
\end{tabular}

$\hat{\imath}, \mathrm{P}<0.01$ compared with the control group.

\subsection{Changes in Plasma Glucose}

12-weeks of therapy decreased the blood pressure and plasma glucose. The combined treatment was a little more effective than treatment with western medicine alone, as shown in table 2.

Table 2. Changes of blood pressure and plasma glucose in the two groups $(\bar{x} \pm s)$.

\begin{tabular}{|c|c|c|c|c|}
\hline & \multicolumn{2}{|l|}{ Treatment group } & \multicolumn{2}{|l|}{ Control group } \\
\hline & Before treatment & After treatment & Before treatment & After treatment \\
\hline $\mathrm{SBP}(\mathrm{mmHg})$ & $148 \pm 14$ & $136 \pm 17^{\text {讼 } \Delta}$ & $145 \pm 14$ & $139 \pm 18$ \\
\hline DBP (mmHg) & $87 \pm 11$ & $78 \pm 12^{2 / \Delta}$ & $88 \pm 14$ & $83 \pm 12$ \\
\hline FPG $(\mathrm{mmol} / \mathrm{L})$ & $8.47 \pm 1.25$ & 6. $77 \pm 1.01^{\grave{\Delta} \Delta}$ & $8.64 \pm 1.33$ & $7.89 \pm 1.56^{\Delta}$ \\
\hline 2-hPG (mmol/L) & $15.48 \pm 3.61$ & $9.27 \pm 2.57^{\grave{\lambda} \Delta}$ & 16. $12 \pm 3.32$ & 10. $12 \pm 2.85$ \\
\hline $\mathrm{Hb}$ Alc $(\%)$ & $9.35 \pm 1.43$ & $7.25 \pm 0.36^{\text {t. } \Delta}$ & $9.46 \pm 1.68$ & $7.89 \pm 1.68^{\Delta}$ \\
\hline
\end{tabular}

$\Delta, \mathrm{P}<0.01$ compared with before treatment; $\downarrow \vec{\jmath}, \mathrm{P}<0.01$ compared with the control group.

\subsection{Changes in Urine Albumin Levels and the Kidney Function}

As indicates of early DN, urine albumin levels decreased obviously in treatment group after treatment. The urine protein/creatinine ratio, B2MU and A1MU decreased noticeably in treatment group after treatment. Compared with the control group, greater decreases were observed in the treatment group. The kidney function, as measured by the eGFR, Cyst, Scr and SUA, significantly improved after treatment, as shown in table 3.

Table 3. The changes in albumin and kidney function $(\bar{x} \pm s)$.

\begin{tabular}{|c|c|c|c|c|}
\hline & \multicolumn{2}{|l|}{ Treatment group } & \multicolumn{2}{|l|}{ Control group } \\
\hline & Before treatment & After treatment & Before treatment & After treatment \\
\hline ALBU $(\mathrm{mg} / \mathrm{L})$ & $2564.6 \pm 45.8$ & $170.8 \pm 4.8^{\text {ì } \Delta}$ & $2489.8 \pm 56.7$ & $1237.8 \pm 87.4^{\Delta}$ \\
\hline $\mathrm{U} / \mathrm{C}(\mathrm{mg} / \mathrm{mmolCr})$ & $12.35 \pm 2.43$ & $6.25 \pm 1.36^{i \Delta \Delta}$ & $12.46 \pm 3.68$ & $10.89 \pm 2.68^{\Delta}$ \\
\hline B2MU (mg/L) & $2.35 \pm 0.43$ & $1.25 \pm 0.36^{\text {究 } \Delta}$ & $2.46 \pm 0.68$ & $1.89 \pm 0.68^{\Delta}$ \\
\hline $\mathrm{A} 1 \mathrm{MU}(\mathrm{mg} / \mathrm{L}$ & $17.35 \pm 2.43$ & $8.25 \pm 2.36^{\text {tr } \Delta}$ & $16.46 \pm 5.68$ & $15.89 \pm 3.68^{\Delta}$ \\
\hline eGFR (ml/min) & $35.44 \pm 9.45$ & 44. $32 \pm 8.66^{\star \Delta \triangle}$ & $36.47 \pm 8.97$ & $37.86 \pm 7.54^{\Delta}$ \\
\hline Cyst $(\mathrm{mg} / \mathrm{L})$ & $2.14 \pm 0.36$ & $0.96 \pm 0.44^{\text {部 }}$ & $2.18 \pm 0.55$ & $1.97 \pm 0.67^{\Delta}$ \\
\hline $\operatorname{Scr}(\mu \mathrm{mol} / \mathrm{L})$ & $132 \pm 16$ & $87 \pm 12^{\text {勏 }}$ & $129 \pm 23$ & $124 \pm 17 \triangle$ \\
\hline $\mathrm{SUA}(\mu \mathrm{mol} / \mathrm{L})$ & $425 \pm 43$ & $348 \pm 36^{\text {in } \Delta}$ & $423 \pm 68$ & $419 \pm 78^{\Delta}$ \\
\hline
\end{tabular}

$\Delta, \mathrm{P}<0.01$ compared with before treatment; 2 , $\mathrm{P}<0.01$ compared with the control group. 


\subsection{Changes in Blood Lipid}

The average total cholesterol and LDL cholesterol levels were significantly decreased after treatment compared with before treatment. Compared with the control group, greater decreases were observed in the treatment group, as shown in table 4 .

Table 4. The changes in lipid and lever function in the two groups $(\bar{x} \pm s)$.

\begin{tabular}{|c|c|c|c|c|}
\hline & \multicolumn{2}{|l|}{ Treatment group } & \multicolumn{2}{|l|}{ Control group } \\
\hline & Before treatment & After treatment & Before treatment & After treatment \\
\hline FFA (mmol/L) & $0.68 \pm 0.12$ & $0.35 \pm 0.13^{i \Delta \Delta}$ & $0.67 \pm 0.9$ & $0.59 \pm 0.11$ \\
\hline $\operatorname{LDLC}(\mathrm{mmol} / \mathrm{L})$ & $5.23 \pm 0.98$ & $3.76 \pm 1.34^{\sharp \Delta}$ & $5.18 \pm 1.45$ & $4.96 \pm 1.21^{\Delta}$ \\
\hline $\mathrm{HDLC}(\mathrm{mmol} / \mathrm{L})$ & $1.12 \pm 0.45$ & $1.45 \pm 0.23^{\text {㐫 } \Delta}$ & $1.19 \pm 0.54$ & $1.24 \pm 0.47^{\Delta}$ \\
\hline $\mathrm{TC}(\mathrm{mmol} / \mathrm{L})$ & $6.14 \pm 1.11$ & $4.87 \pm 1.34^{\wedge \Delta}$ & $6.22 \pm 1.44$ & $5.69 \pm 1.36^{\Delta}$ \\
\hline TRIG (mmol/L) & $2.14 \pm 0.65$ & $1.23 \pm 0.33^{\text {嵌 }}$ & $2.26 \pm 0.77$ & $1.87 \pm 0.88^{\Delta}$ \\
\hline $\operatorname{AST}(\mathrm{u} / \mathrm{L})$ & $34 \pm 7$ & $32 \pm 4$ & $31 \pm 8$ & $32 \pm 4$ \\
\hline $\operatorname{ALT}(\mathrm{u} / \mathrm{L})$ & $35 \pm 8$ & $32 \pm 6$ & $37 \pm 3$ & $36 \pm 9$ \\
\hline $\operatorname{ALB}(\mathrm{g} / \mathrm{L})$ & $35.45 \pm 2.43$ & $56.43 \pm 6.36^{\grave{\lambda} \Delta}$ & $35.86 \pm 7.68$ & $37.35 \pm 9.68^{\Delta}$ \\
\hline
\end{tabular}

$\Delta, \mathrm{P}<0.01$ compared with before treatment; 论, $\mathrm{P}<0.01$ compared with the control group.

\section{5. The Changes in Immunological and Inflammatory Index}

The inflammatory index CRP, TNF- $\alpha$ and MCP-1 levels decreased obviously in treatment group after treatment. Compared with the control group, greater decreases were observed in the treatment group. The Immunological index C3, C4, IgA and IaG increased noticeably in treatment group after treatment, as shown in table 5.

Table 5. The changes in Immunological and inflammatory index $(\bar{x} \pm s)$.

\begin{tabular}{|c|c|c|c|c|}
\hline & \multicolumn{2}{|l|}{ Treatment group } & \multicolumn{2}{|l|}{ Control group } \\
\hline & Before treatment & After treatment & Before treatment & After treatment \\
\hline $\mathrm{CRP}(\mathrm{mg} \mathrm{L})$ & $9.86 \pm 1.31$ & $4.13 \pm 2.18^{17 \Delta}$ & $8.95 \pm 2.35$ & $6.09 \pm 3.65^{\Delta}$ \\
\hline $\mathrm{TNF}-\alpha(\mathrm{ng} \mathrm{L})$ & $24.36 \pm 2.3$ & $13.45 \pm 3.56^{2 \Delta}$ & $23.87 \pm 3.67$ & $21.58 \pm 4.05^{\Delta}$ \\
\hline MCP-1（ng/ml） & $15.32 \pm 4.35$ & $4.56 \pm 2.42^{2} \Delta$ & $15.35 \pm 5.46$ & $13.46 \pm 5.89^{\Delta}$ \\
\hline $\mathrm{C} 3(\mathrm{mg} / \mathrm{L})$ & $756 \pm 56$ & $1024 \pm 68^{\star \Delta}$ & $749 \pm 87$ & $768 \pm 95^{\Delta}$ \\
\hline $\mathrm{C} 4(\mathrm{mg} / \mathrm{L})$ & $122 \pm 32$ & $245 \pm 67^{i>\Delta}$ & $132 \pm 87$ & $142 \pm 78^{\Delta}$ \\
\hline $\operatorname{Ig} \mathrm{A}(\mathrm{g} / \mathrm{L})$ & $0.89 \pm 0.23$ & $2.35 \pm 1.12^{\star \Delta}$ & $0.91 \pm 0.89$ & $1.02 \pm 0.78^{\Delta}$ \\
\hline
\end{tabular}

$\Delta, \mathrm{P}<0.01$ compared with before treatment; $\grave{\hat{\gamma}}, \mathrm{P}<0.01$ compared with the control group.

\subsection{The Changes in The Symptoms of Two Groups}

After 12-weeks of therapy, the symptoms of treatment group improved obviously. There was no obvious change on symptoms of control group, as shown in table 6 .

Table 6. The changes in The symptoms of two groups.

\begin{tabular}{|c|c|c|c|c|}
\hline & \multicolumn{2}{|l|}{ Treatment group } & \multicolumn{2}{|l|}{ Control group } \\
\hline & Before treatment & After treatment & Before treatment & After treatment \\
\hline lassitude & $5.4 \pm 0.78$ & $2.4 \pm 68^{i s}$ & $5.3 \pm 0.89$ & $4.98 \pm 0.65$ \\
\hline shortness of breath & $3.25 \pm 56$ & $1.54 \pm 0.56^{i \vec{\Delta}} \Delta$ & $3.45 \pm 0.65$ & $3.25 \pm 0.67$ \\
\hline dizziness & $3.65 \pm 1.31$ & $2.05 \pm 89^{\text {خ }} \Delta$ & $3.87 \pm 52$ & $3.46 \pm 87$ \\
\hline frequent urination & $5.49 \pm 1.23$ & $2.53 \pm 1.32^{\star \Delta} \Delta$ & $5.32 \pm 1.23$ & $4.89 \pm 1.52$ \\
\hline vertigo & $5.44 \pm 1.15$ & $1.86 \pm 0.66^{\lambda \Delta}$ & $5.51 \pm 1.23$ & $4.98 \pm 0.96$ \\
\hline tinnitus & $3.25 \pm 1.21$ & $1.35 \pm 62^{\text {}} \Delta$ & $3.18 \pm 1.23$ & $3.14 \pm 0.97$ \\
\hline soreness of the knees & $2.88 \pm 66$ & $1.36 \pm 0.45^{\natural \Delta}$ & $2.87 \pm 0.76$ & $2.04 \pm 0.77$ \\
\hline colorless lips and nails & $5.42 \pm 1.21$ & $2.56 \pm 85^{\text {고 }}$ & $5.61 \pm 1.52$ & $4.86 \pm 1.25$ \\
\hline frequent urination & $2.78 \pm 1.15$ & $1.34 \pm 0.55^{\text {r } \triangle}$ & $2.69 \pm 0.44$ & $2.30 \pm 0.88$ \\
\hline long or deficient urine & $2.66 \pm 0.17$ & $1.33 \pm 0.22^{i \Delta}$ & $2.73 \pm 0.84$ & $2.44 \pm 0.68$ \\
\hline nocturia & $2.38 \pm 1.12$ & $1.36 \pm 0.66^{r \Delta}$ & $2.54 \pm 0.78$ & $2.31 \pm 0.89$ \\
\hline diarrhea before dawn & $1.55 \pm 0.42$ & $1.22 \pm 0.81^{\text {tr } \Delta}$ & $1.62 \pm 0.66$ & $1.55 \pm 0.54$ \\
\hline dreaminess & $2.33 \pm 1.22$ & $1.45 \pm 0.61^{1 / \Delta}$ & $2.41 \pm 0.79$ & $2.33 \pm 0.82$ \\
\hline pale face & $3.64 \pm 1.28$ & $1.31 \pm 0.55^{\text {}} \Delta$ & $3.59 \pm 1.08$ & $3.21 \pm 1.24$ \\
\hline
\end{tabular}

$\Delta, \mathrm{P}<0.01$ compared with before treatment; $\downarrow, \mathrm{P}<0.01$ compared with the control group. 


\section{Discussion}

Diabetic nephropathy is a serious microvascular complication of diabetes, is the leading cause of end-stage renal disease. Despite optimal treatment, including glycaemic comtrol and antihypertensive therapy, the disease progresses [18].

Tight glycemic controling can reduce albuminuria and the risk of end-stage renal disease. These encouraging data must be interpreted with caution, as reduction in albuminuria may be offset by the negative consequences of hypoglycemia from strict diabetic control. The ACCORD (Action to Control Cardiovascular Risk in Diabetes) trial was terminated early due to excess mortality in the intensive therapy arm (HbA1c target $<6.0 \%$ ) versus the standard arm (HbAlc 7. 0-7.9\%) [19]. In the UKPDS, patients in the intensive group had significantly more hypoglycemic episodes than those in the conventional group, regardless of whether data were analyzed by intent-to-treat or actual therapy [20]. Severe hypoglycemia observed in the ADVANCE cohort was linked to a range of adverse clinical effects, which prompted speculation on what constitutes optimal diabetic control. The American Association of Clinical Endocrinologists recommends an HbA1c target of $<6.5 \%$, while the American Diabetes Association sets a goal of $\mathrm{HbA} 1 \mathrm{c}<7 \%$, aiming to strike a balance between the risk of hypoglycemia and the clear benefit of renoprotection [21-23]. Our study indicates that combined Chinese medicine and western medicine is more effective than western medicine alone on reducing blood glucose without hypoglycemia.

Hypertension has long been known to be an independent, modifiable variable which predisposes individuals with DM to the development and acceleration of micro- and macro-vascular problems. Prospective observational data from UK Prospective Diabetes Study showed that, for every $10 \mathrm{mmHg}$ reduction in systolic blood pressure, there was a decrease in all DM-related complications and death by $12 \%$ and $15 \%$, espectively blockade of the renin-angiotensin system (RAS) using angiotensinconverting enzyme inhibitors (ACEI) or angiotensin II receptor blockers (ARB) is superior to using other anti-hypertensive agents in DN. The prevention and the treatment with drugs interacting with RAAS are one of the greatest successes of the pharmacological research in the last years. Many trials demonstrated the efficacy of ARBs and ACEI in preventing or reducing the progression of albuminuria, the loss of kidney function and the mortality in diabetic population. They provide other renoprotective benefits beyond simply regulation of blood pressure. For any given level of blood pressure reduction, after 24 weeks valsartan was shown to perform better than amlodipine in reducing micro-albuminuria. But dual blockade of RAAS may bring cardiovascular and renal adverse events, even in presence of a reduction of albuminuria. [24-26].

The role of lipid-lowering treatments in renoprotection for patients with diabetes is debatable. Statins are the most widely used class of drug for lipid lowering in individuals with type 2 diabetes, reflecting the indisputable evidence that lowering of
LDL cholesterol in individuals with type 2 diabetes is associated with reduced cardiovascular events and mortality. The available evidence supports the adjunctive early use of fenofibrate in type 2 diabetes mellitus for the prevention of microvascular complications, particularly in individuals presenting with the first signs of the complication and during the initial stages of the disease [27]. Our results suggests Chinese medicine can reduce lipid in some degree.

Although the exact cause of DN remains unclear, several mechanisms have been postulated, such as hyperglycemia-induced renal hyper filtration and renal injury, AGEs-induced increased oxidative stress, activated PKC-induced increased production of cytokines, chemokines, and different inflammatory and apoptotic signals. Some study demonstrated that oral high-dose vitamin E supplementation for 12 weeks among DN patients had favorable effects on biomarkers of kidney injury, inflammation, and oxidative stress. Kremezin and benfotiamine are AGEs inhibitors, another therapeutic target against DN. Ruboxistaurin, rapamycin, aliskiren, and manidipine are some FDA approved pharmacotherapeutics effective against $\mathrm{DN}$ via diverse mechanisms [28, 29]. Amiloride increased renal $\mathrm{Na}$ excretion, reduced blood pressure, albuminuria, and total and active plasmin in urine. It is concluded that epithelial sodium channel is an attractive target to attain blood pressure control in long-term type I diabetes with no enhanced activity associated with nephropathy [30]. As a selective endothelin A receptor antagonist, Atrasentan has been shown to reduce albuminuria in type 2 diabetes. It protects endothelial function and tissue homeostasis through the antialbuminuric effects [31].

Despite the medications available in the market to treat DN, the involvement of multiple mechanisms makes it difficult to choose an optimum therapeutic agent. Therefore, much research is required to find out new therapeutic agent/strategies for an adequate pharmacotherapy of DN.

Chinese medicine is a good choice for patients with DN. Moutan Cortex terpene glycoside ameliorats endoplasmic reticulum stress-related inflammation in the pathogenesis of $\mathrm{DN}$, wherein the protective mechanism might be associated with the inhibition of IRE1/NF- $\kappa$ B activation. It might be a potential therapeutic candidate for the prevention and treatment of DN [32]. Green tea polyphenols administration reduces albuminuria in diabetic patients receiving the maximum recommended dose of renin-angiotensin. Reduction in podocyte apoptosis by activation of the WNT pathway may have contributed to this effect [33]. Tongxinluo protects podocyte from apoptosis in DN, partially through its antioxidant effect and inhibiting of the activation of P38 and caspase-3 [34].

\section{Conclusion}

The results indicate that combined therapy with Chinese medicine and western is more effective than Chinese medicine or western alone, especialy on improving symptoms of patients with DN. QZD may protect DN through decrease lipid and inflammation. 


\section{Acknowledgements}

This work is supported by grants from Guangdong Provincial Natural Science (S2012010010783), and Guangdong Provincial Administration of Traditional Chinese Medicine (20121261).

\section{Disclosure of Conflict of Interest}

There is no conflict of interest.

\section{References}

[1] Martínez-Castelao A, Navarro-González JF, Górriz JL, de Alvaro F. The Concept and the Epidemiology of Diabetic Nephropathy Have Changed in Recent Years. J Clin Med. 2015; 4 (6):1207-1216.

[2] Farag YM, Gaballa MR. Diabesity: an overview of a rising epidemic. Nephrol Dial Transplant. 2011; (1):28-35.

[3] Gaballa M, Farag MK. Predictors of Diabetic Nephropathy. Eur J Med. 2013; 8:287-296.

[4] Ziamajidi N, Nasiri A, Abbasalipourkabir R, Sadeghi Moheb S. Effects of garlic extract on TNF- $\alpha$ expression and oxidative stress status in the kidneys of rats with STZ + nicotinamide-induced diabetes. Pharm Biol. 2017, 55 (1):526-531.

[5] Katsoulieris EN, Drossopoulou GI, Kotsopoulou ES, Vlahakos DV, Lianos EA, Tsilibary EC. High Glucose Impairs Insulin Signaling in the Glomerulus: An In Vitro and Ex Vivo Approach. PLoS One. 2016 Jul 19; 11 (7):e0158873.

[6] Jo HA, Kim JY, Yang SH, Han SS, Joo KW, Kim YS, Kim DK. The role of local IL6/JAK2/STAT3 signaling in high glucose-induced podocyte hypertrophy. Kidney Res Clin Pract. 2016, 35 (4):212-218.

[7] Zhang Y, Xiao HQ, Zeng XT, Zuo HX, Xu YC. Associations between endothelial nitric oxide synthase polymorphisms and risk of diabetic nephropathy: an updated meta-analysis. Ren Fail. 2015, 37 (10):312-326.

[8] Hanning You; Ting Gao; Timothy K. Cooper; W. Brian Reeves; Alaa S. Awad. Macrophages directly mediate diabetic renal injury. American Journal of Physiology Renal Physiology, 2013, 305, (12):, F1719-F1727

[9] Mir M, Rostami A, Hormozi M. Comparison of serum levels of IL-18 in peripheral blood of patients with type II diabetes with nephropathyclinical protests and patients with type II diabetes without nephropathy clinical protests. Diabetes Metab Syndr. 2016; 31 (16):30171-30180.

[10] Harshini Mudaliar; Carol Pollock; Muralikrishna Gangadharan Komala; Steven Chadban; Huiling Wu; Usha Panchapakesan. The role of Toll-like receptor proteins (TLR) 2 and 4 in mediating inflammation in proximal tubules. American Journal of Physiology Renal Physiology. 2013, 305, (12):F143-F154

[11] Molinario R, Pocino K, Daloiso PD, Giannace A, Spirito G, Zuppi C, Antenucci M. Urinary Albumin Detection: Comparison of Two Different Methods. J Clin Lab Anal. 2016; 30 (6):888-891.
[12] Said SM1, Nasr SH2. Silent diabetic nephropathy. Kidney Int. 2016; 90 (1):24-26.

[13] Pan Y, Jiang S, Qiu D, Shi J, Zhou M, An Y, Ge Y, Xie H, Liu Z. Comparing the GFR estimation equations using both creatinine and cystatin $\mathrm{c}$ to predict the long-term renal outcome in type 2 diabetic nephropathy patients. J Diabetes Complications. 2016, S1056-8727 (16):30292-6.

[14] Xuan L, Zheng F, Lin X, Zhu W, Yin X, Li H. Correlation between serum free fatty acid level and estimated glomerular filtration rate in type 2 diabeticpatients. Zhonghua Yi Xue Za Zhi. 2016; 96 (17):1320-1324.

[15] Brenneman J, Hill J, Pullen S. Emerging therapeutics for the treatment of diabetic nephropathy. Bioorg Med Chem Lett. 2016, 15; 26 (18):4394-402.

[16] Chamberlain JJ, Rhinehart AS, Shaefer CF Jr, Neuman A. Diagnosis and Management of Diabetes: Synopsis of the 2016 American Diabetes AssociationStandards of Medical Care in Diabetes. Ann Intern Med. 2016; 164 (8):542-552.

[17] Zheng YY: Guidelines for Clinical Research of Chinese Medicine (New Drug). Beijing: Chinese Medicine and Science Publication House; 2002.

[18] Pofi R, Di Mario F, Gigante A, Rosato E, Isidori AM, Amoroso A, Cianci R, Barbano B. Diabetic Nephropathy: Focus on Current and Future Therapeutic Strategies. Curr Drug Metab. 2016; 17 (5):497-502.

[19] Papademetriou V, Lovato L, Tsioufis C, Cushman W, Applegate WB, Mottle A, Punthakee Z, Nylen E, Doumas M; ACCORD Study Group. Effects of High Density Lipoprotein Raising Therapies on Cardiovascular Outcomes in Patients with Type 2 Diabetes Mellitus, with or without Renal Impairment: The Action to ControlCardiovascular Risk in Diabetes Study. Am J Nephrol. 2016; 45 (2):136-145.

[20] Bonora E, Bryzinski B, Hirshberg B, Cook W. A post hoc analysis of saxagliptin efficacy and safety in patients with type 2 diabetes stratified byUKPDS 10-year cardiovascular risk score. Nutr Metab Cardiovasc Dis. 2016; 26 (5):374-379

[21] Mohammedi K, Woodward M, Hirakawa Y, Zoungas S, Williams B, Lisheng L, Rodgers A, Mancia G, Neal B, Harrap S, Marre M, Chalmers J; ADVANCE Collaborative Group. Microvascular and Macrovascular Disease and Risk for Major Peripheral Arterial Disease in Patients With Type 2 Diabetes. Diabetes Care. 2016; 39 (10):1796-1803.

[22] Helou N, Talhouedec D, Shaha M, Zanchi A. The impact of a multidisciplinary self-care management program on quality of life, self-care, adherence to anti-hypertensive therapy, glycemic control, and renal function in diabetic kidney disease: A Cross-over Study Protocol. BMC Nephrol. 2016; 17 (1):88.

[23] Górriz JL, Nieto J, Navarro-González JF, Molina P, Martínez-Castelao A, Pallardó LM. Nephroprotection by Hypoglycemic Agents: Do We Have Supporting Data?J Clin Med. 2015;4 (10):1866-1889.

[24] Persson F, Theilade S, Eugen-Olsen J, Rossing P, Parving HH. Renin angiotensin system blockade reduces urinary levels of soluble urokinaseplasminogen activator receptor (suPAR) in patients with type 2 diabetes. J Diabetes Complications. 2016; S1056-8727 (16) 30282-3. 
[25] Grolla E, Bonanni L, Cutolo A, Presotto F, Dalla Vestra M. Disputes in the Treatment of Diabetic Nephropathy: The Dual Blockade of Renin-Angiotensin System. Exp Clin Endocrinol Diabetes. 2016; 124 (6):361-6.

[26] Adler AI, Stratton IM, Neil HA, et al.: Association of systolic blood pressure with macrovascular and microvascular complications of type 2 diabetes (UKPDS 36): prospective observational study. BMJ. 2000; 321 (7258): 412-419.

[27] Czupryniak L, Joshi SR, Gogtay JA, Lopez M. Effect of micronized fenofibrate on microvascular complications of type 2 diabetes: a systematic review. Expert Opin Pharmacother. 2016 Aug; 17 (11):1463-1473.

[28] Bhattacharjee N, Barma S, Konwar N, Dewanjee S, Manna P. Mechanistic insight of diabetic nephropathy and its pharmacotherapeutic targets: An update. Eur J Pharmacol. $2016 ; 25 ; 791: 8-24$.

[29] Khatami PG, Soleimani A, Sharifi N, Aghadavod E, Asemi Z. The effects of high-dose vitamin $\mathrm{E}$ supplementation on biomarkers of kidney injury, inflammation, and oxidative stress in patients with diabetic nephropathy: A randomized, double-blind, placebo-controlled trial. J Clin Lipidol. 2016;10 (4):922-929.

[30] Andersen H, Hansen PB, Bistrup C, Nielsen F, Henriksen JE, Jensen BL. Significant natriuretic and antihypertensive action of the epithelial sodium channel blocker amiloride indiabetic patients with and without nephropathy. J Hypertens. 2016;34 (8):1621-1629.

[31] Boels MG, Avramut MC, Koudijs A, Dane MJ, Lee DH, van der Vlag J, Koster AJ, van Zonneveld AJ, van Faassen E, Gröne HJ, van den Berg BM, Rabelink TJ. Atrasentan Reduces Albuminuria by Restoring the Glomerular Endothelial Glycocalyx Barrier in Diabetic Nephropathy. Diabetes. 2016; 65 (8):2429-2439.

[32] Chen J, Hou XF, Wang G, Zhong QX, Liu Y, Qiu HH, Yang N, Gu JF, Wang CF, Zhang L, Song J, Huang LQ, Jia XB, Zhang $\mathrm{MH}$, Feng L. Terpene glycoside component from Moutan Cortex ameliorates diabetic nephropathy by regulating endoplasmic reticulum stress-related inflammatory responses. J Ethnopharmacol. 2016; S0378-8741 (16) 30933-3.

[33] Borges CM, Papadimitriou A, Duarte DA, Lopes de Faria JM, Lopes de Faria JB. The use of green tea polyphenols for treating residual albuminuria in diabetic nephropathy: A double-blind randomised clinical trial. Sci Rep. 2016; $20 ; 6: 28282$.

[34] Cui F, Gao Y, Zhao W, Zou D, Zhu Z, Wu X, Tian N, Wang X, Liu J, Tong Y. Effect of Tongxinluo on Podocyte Apoptosis via Inhibition of Oxidative Stress and P38 Pathway in DiabeticRats. Evid Based Complement Alternat Med. 2016; 2016:5957423. 